\title{
Über Restitutionserscheinungen an Stelle von Augen bei Tenebrio-Larven nach Zerstörung der optischen Ganglien.
}

\author{
Von \\ Jar. Kr̛̀iženecký, \\ Prag-Kgl. Weinberge. \\ Mit Tafel XVII.
}

Eingegangen am 14. Juli 1913.

Vor Jahren wurde von C. Hersst in einer Reihe von Arbeiten (siehe Literaturverzeichnis) bewiesen, daB bei den podophthalmen Crustaceen nach der Zerstörung der optischen Ganglien an Stelle von Augen antennenähnliche Heteromorphosen regeneriert werden. Um zu prüfen, ob auch den Insekten eine solche Fähigkeit zukommt, unternahm Dr. VIKTOR JANDA in der Biologischen Versuchsanstalt in Wien eine Reihe von ähnlichen Experimenten an Stylopyga orientalis und an Tenebrio molitor. Nach der Amputation der Augen zerstörte er den Larven der genannten Insekten durch das Ausbrennen der Wunde die optischen Ganglien und bekam an ihrer Stelle fühlerähnliche Regenerate. Obzwar seine Resultate noch geringe sind, geht doch aus ihnen hervor, daB auch bei den Insekten die Restitution der Augen in einer Abhängigkeit von den optischen Ganglien stehen wird. Herr Dr. V. JANDA hatte mir die Erfolge seiner Experimente schon im Februar 1912 mitgeteilt, mit der Aufforderung, diese fortzusetzen. Ich folgte ihm, und die Resultate, zu welchen ich während meiner Experimente gekommen bin, bestätigten und ergänzten in manchem seine Angabe, welche er unterdessen im Arch. f. Entw.Mech. (Bd. 36, S. 1-3) publiziert hatte. Nach JaNDAs Publikation meine ich, daß es nicht ohne Interesse sein wird, auch tiber meine Experimente Mitteilung zu machen.

$\mathrm{Zu}$ den Versuchen benutzte ich mittelalte Larven von Tenebrio 
molitor, welche sich vor der Verpuppung sicher noch drei oder viermal abhäuten mußten. Entgegen der Jandaschen Methode operierte ieh die „Tiere ohne vorhergehender Narkose and zerstörte ihnen die Augen direkt mit den optischen Ganglien mittels einer heißen Nadel. Diese Operation geschah immer an der rechten Seite. Die Augen kommen bei den Tenebrio-Larven als kleine, schwärzliche Punkte zur Seite des Kopfes vor, wie uns dies Fig. 5 (au) darstellt. Weil sie sich sehr nahe bei den Fühlern befinden, so geschieht es leicht, daß bei ihrer Ausbrennung auch die Fühler zerstört werden können. Es wurden also bei diesen Experimenten, außer den Augen mit den Ganglien, auch die Fühler entfernt.

Auf diese Weise operierte ich zwei Serien von Tenebrio-Larven zu je 50 Exemplaren. Ihre weitere Zuichtung geschah in kleineren, mit Kleie und Mehl angefüllten Gefäßen. Dabei hatte sich die Wunde fast bei jedem Tiere durch Pilze, vielleicht Pennicillium glaucum, infiziert und die Folge dieser Infizierung war, daß ein großer Teil der Augenumgebung, manchmal auch mit dem rechten Kiefer, zerstört wurde und bei der folgenden Abhäutung abfiel. Damit näherte sich meine Operation der $\mathrm{J}_{\text {ANDaschen }}$ und ist zugleich der Operation ähnlich, welche I. E. WERBER schon vor Jahren (1905) auch an Tenebrio ausgefuhrt hatte. Diese Ähnlichkeit wird noch im weiteren Besprechung finden.

In beiden Serien gingen die Larven nach der Operation schnell zugrunde und nur wenige von ihnen haben sich einmal abgehäntet. Auch jene, welche die Operation überlebten, schienen in ihrer Lebenstätigkeit gehindert zu sein: ihre Bewegungen waren sehr langsam, sie nahmen sehr wenig Nahrung an, bekamen bald eine plattförmige Gestalt, und was das auffallendste war, ihre erste Häutungsperiode (nach der Operation) hat sich recht verlängert. Obzwar bei den mittelalten Tenebrio-Larven die einzelnen Abhäutungen normalerweise in monatlichen Perioden, welche durch Entfernung eines Organes fast immer verkürzt sein können, nacheinander folgen, dauerte es hier bei meinen Experimenten immer mehr als 1 Monat und manchmal auch 2 Monate, bis sich die operierten Tiere zum erstenmal abgehäutet hatten. Die weiteren Häutungen, soviel mir zu beobachten möglich war, gingen dann wieder normalerweise in monatlichen Perioden nacheinander vor sich.

Die Sterblichkeit war bei beiden Serien recht groß, so daß von allen 100 operierten Larven nur vier am Leben geblieben waren und für uns charakteristische Regenerate ausgebildet haben. 
Nach diesen, die allgemeine Seite meiner Experimente betreffenden Vorbemerkungen können wir nun an die eigentliche Beschreibung der Resultate herantreten.

Die erste Serie von 50 Larven operierte ich am 11. II. 12. Viele Larven gingen gleich nach der Operation zugrunde, die übrigen nach der ersten Häntung, nach welcher noch kein Regenerat an der Wunde zu bemerken war; die Wunde war nur mittels eines weichen, unpigmentierten Häutchens vernarbt. Sich zum zweitenmal abzuhäuten und Regenerate auszubilden, gelang nur zwei Larven, welche ich im weiteren genauer beschreiben werde.

Die erste war aus den sich am frühesten zum erstenmal abgehäuteten Larven - sie passierte die erste Abhäutang am 24. III. Die zweite Abhäutung erfolgte am 3. IV. Nach dieser kam schon ein Regenerat zutage. Die Wunde war vernarbt und durch ein weiches, helles Häutchen bedeckt. Das Auge wie auch die Antenne fehlten total und an ihrer Stelle erschien ein starker, zur Seite eingebogener Auswuchs, welcher mit zwei stumpfen Spitzen endete; er entbehrte jeder Borsten, war ganz glatt und ohne Gliederung (vgl. Fig. 1, hm). Am 2. V. hat sich diese Larve in eine Puppe verwandelt, bei welcher das beschriebene Regenerat fast unverändert blieb; auch hier fand ich keine Spur vom Auge. Ein Imago bekam ich nicht, weil die Puppe leider bald zugrunde ging.

Der zweite Fall betraf eine Larve, bei welcher die Pilzinfizierang der Wunde zur Zerstörung eines großen Teiles des Kopfes führte. Dabei wurde auch der rechte Kiefer entfernt and der rechte Vorderteil des Kopfes stark mißgebildet (vgl. Fig. 2). Nach drei Abhäutangen, welche am 9. IV., 5. V. und 16. VI. erfolgten, kam an der Wunde eine Neubildung zutage, der Kiefer regenerierte aber nicht. Auch hier bildete sich an der Stelle des Auges und der Antenne ein Auswuchs, welcher aber, entgegen dem ersten Falle, mit vielen kleinen Borsten bedeckt und in zwei plattförmige, horizontal gelegene, lappenartige Teile zerspaltet war. Die obere Hälfte (Fig. 2, $o b)$, welche an ihrer Basis schmäler als die untere war und an dieser eingewachsen zu sein schien, war weich, fast pigmentlos und stark beweglich, die untere (unt) wieder hart, pigmentiert und fast unbeweglich. - - Aus dieser Larve bekam ich keine Puppe, weil sie bald nach der dritten (nach der Operation) Abhäutung einging.

Am 10. IV. operierte ich eine neue Serie von 50 Larven, von welchen wieder nur zwei am Leben geblieben sind und Regenerate ausgebildet haben. 
Die erste häutete sich am 6. V. ab. Durch die Operation wurde ihr das Auge wie auch die Antenne entfernt; an der Stelle von diesen bildete sich an der durch ein weiches, helles Häutchen vernarbten Wunde ein runder, ungegliederter Auswuchs, welcher viel kleiner als die Auswüchse in den vorhergehenden Fällen war, was dadurch erklärlich ist, daß er schon nach der ersten Häutung (nach der Operation) zutage kam. Dieser Auswuchs (vgl. Fig. 3) war ganz glatt und ohne jede kleinste Borste. Auch diese Larve ging bald zugrunde, ohne das Regenerat weiter ausbilden und differenzieren zu können.

Bei der zweiten Larve aus dieser Serie, welche sich am 12. VI. zum erstenmal nach der Operation abgehäutet hatte, bildete sich an der Stelle des entfernten Auges und der Antenne ein Auswuchs, dessen Form man am besten aus der Abbildung erkennen wird (Fig. 4, hm). Dieser Auswuchs war dem im ersten Falle sehr ähnlich; er war ganz glatt, ohne Borsten, pigmentlos und zugleich unbeweglich. Auch bestand er aus zwei Hälften: die eine war breit mit stumpfen Ecken und die sich anschließende zweite Hälfte war kleiner, zugespitzt, und an ihrem Ende trug sie ein kleines Glied (Fig. $4 a, a m$ ). Auch von dieser Larve gelang es mir nicht, weitere Entwicklungsstadien zu bekommen, weil sie bald nach der ersten Häutung einging.

In allen vier Fällen wurden mit den Augen auch die optischen Ganglien zerstört. Die Augen regenerierten nicht und auch die mit ihnen entfernten Fihler nicht, sondern an Stelle dieser beiden Organe bildeten sich größere Auswüchse, welche den Charakter der Tastorgane nicht entbehrten; besonders klar war dies im zweiten Falle, in welchem man die reichen, kleinen Borsten mit großer Wahrscheinlichkeit als Tastborsten bezeichnen kann. Der kleine Anlauf zur Gliederung im vierten Falle (Fig. 4, $h m$ und Fig. $4 a$ ) läßt mehr als bloß ahnen, daß die Auswtichse mit den Fthlern zusammenhängen. Und doch kann man sie nicht für Regenerate der bloßen Futhler halten, weil sie zu groß sind. Da aber zugleich die Angen, welchen die optischen Ganglien zerstört warden, fehlen, so bin ich der Meinung, daß es sich hier umeine Zusammenbindung der Regenerate der Fuhler und der Restitutionsbildungen an Stelle von Augen handelt; und ich meine auch, daß man nicht fehlgehen wird, wenn man direkt von einer Zusammenbindung beider Nerven, der Augen- und Fuhlernerven, sprechen wird. 
Daß man die Ursache der Entwicklung der beschriebenen Heteromorphosen in der Zerstörung der optischen Ganglien und nicht in der Amputation des Kopfteiles mit dem Auge und der Antenne suchen kann, dafür spricht eine ältere Angabe Werbers (1905): Dieser Forscher amputierte auch die Umgebung vom Ange bei der Tenebrio-Larve mit der Antenne, und zwar durch eine radikale Operation, aber die optischen Ganglien zerstörte er nicht; die Folge dieser Operation war, daß das Auge wie auch die Antenne normal regeneriert haben. Direkt kann ich die Zusammenbindung von Fuhler- und Augennerven nicht beweisen, weil mir mikroskopische Präparate nicht zu Gebote stehen; vielleicht wird dies einmal jemandem anderen gelingen. Für jetzt geht aus meinen Experimenten folgendes Faktum hervor: In vier Fällen, in welchen ich die optischen Ganglien mit den Augen zerstört und zugleich Fubler entfernt habe, kamen an ihren Stellen Ausbildungen zutage, welche ganz klar den Charakter von Tastorganen zeigten.

Zam Schlusse möchte ich noch auf eine interessante Seite des Banes der beschriebenen Ausbildungen hinzeigen.

Betrachtet man die vier oben beschriebenen Erscheinungen zasammen, so sehen wir, daß sie, entgegen dem Jandaschen Befunde, viel komplizierterer Natur sind. In unsern Fällen wurde das Auge mit der Antenne entfernt und anstatt dieser zwei Organe produzierte sich immer eine Neubildung. In den drei, in der Differenzierung weiter fortgeschrittenen Fällen war aber diese Neubildung in zwei Hälften geteilt: entweder war diese Zerteilung in der horizontalen Richtung (Fig. 1 und 4) oder vertikalen (Fig. 2). In den Fällen der horizontalen Zerteilung waren beide Neubildungen glatt, fast unbeweglich, ohne Borsten und unpigmentiert. In dem Falle der vertikalen Zerteilung waren beide Hälften stark mit Borsten bedeckt, eine war unbeweglich aber pigmentiert, die zweite beweglich, aber fast pigmentlos. Es scheint hier also eine Regel in der Aufbauung der Regenerate zu herrschen; ich will aus diesen Umständen zwar keine Konsequenzen ziehen, weil dies in meinen wenigen Fällen vielleicht ein bloBer Zufall war, doch darf man dies anch nicht ubersehen und deswegen mache ich anf diese Erscheinung für jetzt aufmerksam.

Prag-Kgl. Weinberge, Anfang Juli 1913. 
634 Jar. Křiženecký, Über Restitutionserscheinungen an Stelle von Augen nsw.

\section{Literaturverzeichnis.}

Herbst, C., Über Regeneration von antennenähnlichen Organen an Stelle von Augen. 1., 3., 4. u. 5. Mitteilung. Arch. f. Entw.-Mech. Bd. 2, 9 u. 13. 1896, 1900 u. 1901

JANDA, V., Fühlerähnliche Heteromorphosen an Stelle von Augen bei Stylopyga orientalis und Tenebrio molitor. (Experimentelle Studie.) Arch. f. Entw.Mech. Bd. 36. 1913.

Przibram, H., Experimental-Zoologie. 2. Regeneration. Leipzig u. Wien, Deuticke, 1909.

Die Homoeosis bei Arthropoden. Arch. f. Entw.-Mech. Bd. 29.1910.

Werber, I. E., Regeneration des exstirpierten Fühlers und Auges beim Mehlkäfer (Tenebrio molitor). Arch. f. Entw.-Mech. Bd.19. 1905.

\section{Erklärung der Abbildungen.}

(Alle Abbildungen sind vergrößert.)

\section{Tafel XVII.}

Fig. 1. Der Kopf von einer Tenebrio-Larve nach der zweiten (nach der Operation) Abhäutung. An Stelle des entfernten Auges and Fühlers bildete sich nach Zerstörung der optischen Ganglien ein ovaler, glatter und ungegliederter Answuchs $(\mathrm{hm})$, welcher mit zwei stumpfen Spitzen endete $(I, I I)$. Der Fühler der andern Seite $(f)$ und die beiden Kiefer $(m)$. blieben nnverändert.

Fig. 2. Der Kopf einer andern Larve nach derselben Operation. Der rechte Kiefer fehlt und an Stelle des Auges und Fühlers findet man einen mit Borsten bedeckten Auswuchs ( $h m$ ), welcher in zwei Hälften geteilt ist: die obere $(o b)$ ist schmäler, ohne Pigment und beweglich, die untere (unt) pigmentiert und unbeweglich.

Fig. 3. Der Kopf von einer dritten Larve, bei welcher an Stelle von Fühler und Auge ein kleiner, glatter und heller Auswuchs $(\mathrm{hm})$ regeneriert hat. Die ubrigen Organe des Kopfes (Fühler $=f$ und Kiefer $=m$ ) sind normal geblieben.

Fig. 4. Der Kopf von einer vierten auf erwähnte Weise operierten Larve. An Stelle von Fühler und Auge bildete sich ein zweiteiliger, glatter, unbeweglicher Auswuchs $(\mathrm{hm})$, dessen Form uns Fig. $4 a$ klarer darstellt. Die eine Hälfte dieses Auswuchses (die kleinere) trägt an ihrem Ende einen gliederförmigen Ansatz $(a n)$.

Fig. 5. Ein normaler Kopf einer Tenebrio-Larve von der rechten Vorderseite. Die Bezeichnungen bedeuten : $a u=$ das Auge, $f=$ Fühler, $m=$ die Kiefer, $v b=$ das Vorderbein. 


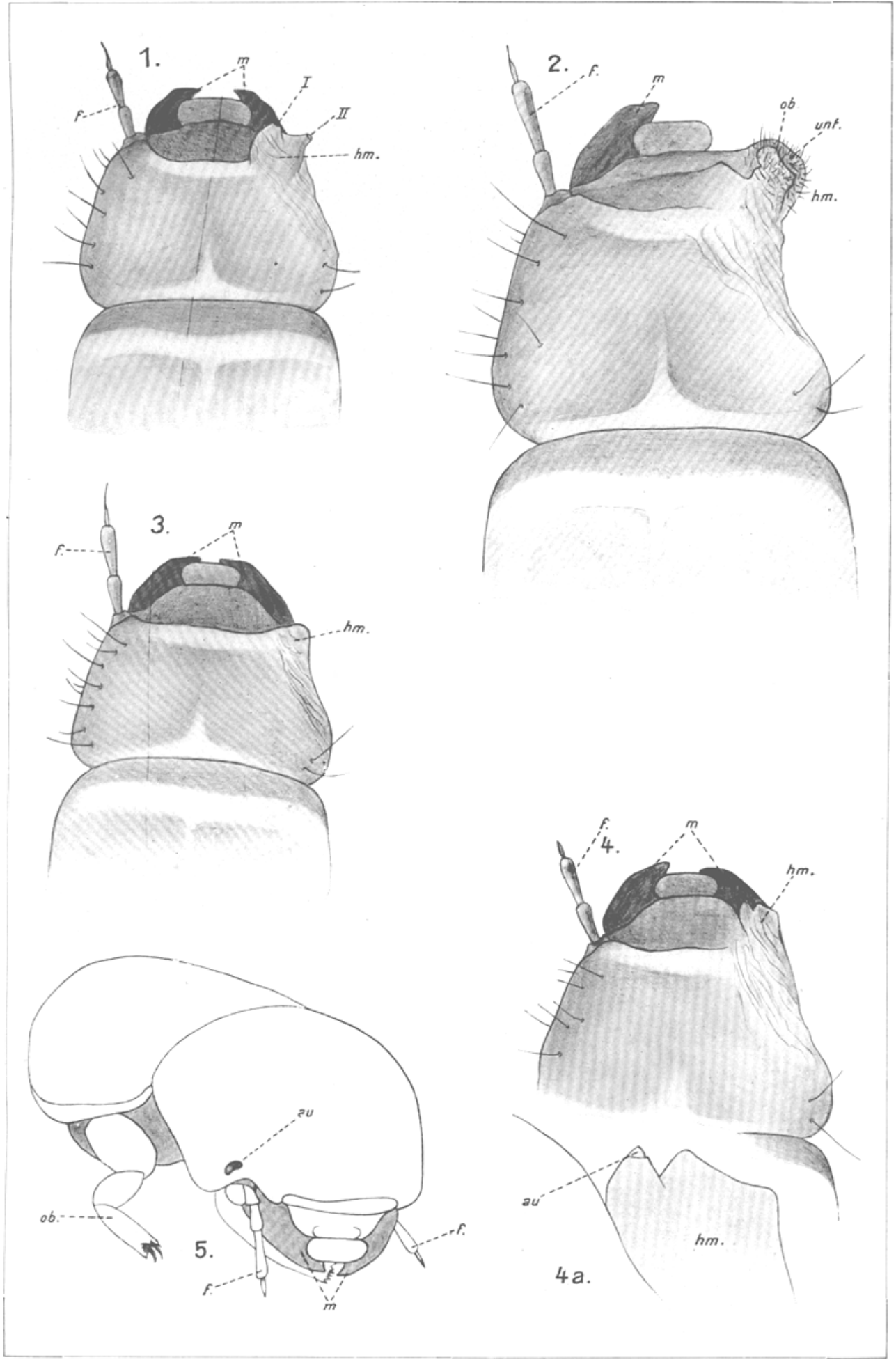

Kr̀iženeckṣ. $\quad$ Verlag von Wilhelm Engelmann in Leipzig und Berlin. 